\title{
Lymphogranulomatose vénérienne
}

\author{
Eric J. Eckbo MD, Malcolm Hedgcock MD, Troy Grennan MD MSc
}

Citation : CMAJ 2021 December 13;193:E1889. doi : 10.1503/cmaj.210853-f

Voir la version anglaise de l'article ici : www.cmaj.ca/lookup/doi/10.1503/cmaj.210853

\footnotetext{
1 La lymphogranulomatose vénérienne (LGV) est une infection agressive, transmissible

1 sexuellement, causée par des souches particulières de Chlamydia trachomatis

Les souches L1, L2 et L3 sont plus invasives que celles causant la plupart des infections anogénitales ${ }^{1-4}$. Avant 2004, on observait rarement la lymphogranulomatose vénérienne au Canada, mais depuis, elle est devenue endémique. Entre 2013 et 2020, 2052 isolats de C. trachomatis transmis au Laboratoire national de microbiologie se sont révélés positifs aux souches bactériennes causant la lymphogranulomatose vénérienne ( $D^{r}$ Alberto Severini, Laboratoire national de microbiologie, Winnipeg : communication personnelle, 2021). Des éclosions se poursuivent en Europe de l'Ouest et en Amérique du Nord, touchant principalement des hommes ayant des relations sexuelles avec d'autres hommes ${ }^{2-6}$.
}

La lymphogranulomatose vénérienne peut présenter un tableau clinique variable qui peut être mal diagnostiqué

Bien que la lymphogranulomatose vénérienne puisse être asymptomatique, le tableau clinique le plus fréquent est un syndrome de rectite où une inoculation anale directe résulte en une rectite hémorragique douloureuse, ressemblant souvent à une maladie inflammatoire de l'intestin ${ }^{1-6}$. Dans le cas d'un syndrome inguinal, par contre, l'infection s'amorce habituellement par une papule indolore et progresse par la suite en une ulcération et une lymphadénopathie inguinale ${ }^{1,4}$. Des symptômes systémiques, comme de la fièvre, des malaises et une arthralgie, sont souvent présents ${ }^{2-6}$.

Les personnes dont les symptômes s'apparentent à une lymphogranulomatose vénérienne ou qui présentent des facteurs de risque devraient procéder à un dépistage de la bactérie

C. trachomatis au moyen d'un test d'amplification des acides nucléiques (TAAN)

On doit insérer des écouvillons à 2 ou $3 \mathrm{~cm}$ à l'intérieur du canal anal; alternativement, des écouvillons peuvent être collectés par visualisation directe lors d'une anuscopie. On peut aussi examiner des aspirats de nœud lymphoïde et des écouvillonnages de lésions génitales suspectes par essai d'amplification de l'acide nucléique. Dans plusieurs centres canadiens, des écouvillonnages rectaux positifs à la bactérie $C$. trachomatis subiront automatiquement des analyses additionnelles pour déterminer le sérotype de la lymphogranulomatose vénérienne.

1 Le traitement contre la lymphogranulomatose vénérienne est de plus longue durée que ceux réservés à d'autres formes de chlamydia

Les lignes directrices canadiennes ${ }^{1}$ recommandent de la doxycycline sous forme orale (100 mg, 2 fois par jour) pour 21 jours comme traitement de première intention. On devrait procéder à un contrôle bactériologique 3 semaines après la fin du traitement. Si on ne la traite pas, la lymphogranulomatose vénérienne peut entraîner des destructions tissulaires irréversibles, des scarifications, des fistules et des obstructions lymphatiques ${ }^{1-6}$.

Les partenaires sexuels d'une personne diagnostiquée devraient être empiriquement traités avec le même schéma thérapeutique que celui de cette personne, en attendant les résultats du test

Tous les partenaires sexuels des 60 derniers jours d'un patient présentant une apparition des symptômes devraient aussi être dépistés pour la lymphogranulomatose vénérienne; ceux qui présentent un résultat positif nécessitent un suivi clinique additionnel, dont un contrôle bactériologique et une enquête de la santé publique. 


\section{Références}

1. Canadian guidelines on sexually transmitted infections. Ottawa: Public Health Agency of Canada; 2020. Accessible ici : https://www.canada.ca/en/public-health/ services/infectious-diseases/sexual-health-sexually-transmitted-infections/canadian-guidelines/chlamydia-lgv.html (consulté le 4 janv. 2021).

2. Weiss E, Sano M. Proctocolitis caused by lymphogranuloma venereum. CMAJ 2018;190:E331-3.

3. Williamson DA, Chen MY. Emerging and reemerging sexually transmitted infections. N Engl J Med 2020;382:2023-32.

4. Mabey D, Peeling RW. Lymphogranuloma venereum. Sex Transm Infect 2002;78:90-2.

5. Boutin CA, Venne S, Fiset M, et al. Lymphogranuloma venereum in Quebec: re-emergence among men who have sex with men. Can Commun Dis Rep 2018; 44:55-61.

6. Cole MJ, Field N, Pitt R, et al. Substantial underdiagnosis of lymphogranuloma venereum in men who have sex with men in Europe: preliminary findings from a multicentre surveillance pilot. Sex Transm Infect 2020;96:137-42.

Intérêts concurrents : Troy Grennan signale un financement institutionnel de la part des laboratoires Merck et Gilead, indépendamment des travaux soumis. Aucun autre intérêt concurrent n'a été déclaré.

Cet article a été révisé par des pairs.

Affiliations : Régie de la santé du littoral de Vancouver, Division de microbiologie médicale et de contrôle des infections (Eckbo), Université de la Colombie-Britannique, Faculté de médecine, Département de pathologie et de médecine de laboratoire (Eckbo); Clinique Spectrum Health (Hedgcock); Centre de contrôle des maladies de la Colombie-Britannique, Services des infections transmissibles sexuellement et du VIH (Grennan); Université de la Colombie-Britannique, Faculté de médecine, Division des maladies infectieuses (Grennan), Vancouver, C.-B.
Propriété intellectuelle du contenu : Il s'agit d'un article en libre accès distribué conformément aux modalités de la licence Creative Commons Attribution (CC BY-NC-ND 4.0), qui permet l'utilisation, la diffusion et la reproduction de tout médium à la condition que la publication originale soit adéquatement citée, que l'utilisation se fasse à des fins non commerciales (c.-à-d., recherche ou éducation) et qu'aucune modification ni adaptation n'y soit apportée. Voir : https://creativecommons.org/ licenses/by-nc-nd/4.0/deed.fr.

Remerciements : Les auteurs remercient le $D^{r}$ Alberto Severini et le personnel du programme d'exanthème viral et des infections transmissibles sexuellement du Laboratoire national de microbiologie pour l'accès aux données nationales de dépistage.

Correspondance : Eric Eckbo, eric.eckbo@vch.ca

Le JAMC vous invite à soumettre vos textes pour la rubrique « Cinq choses à savoir ... » en ligne à http://mc.manuscriptcentral.com/cmaj. 